\title{
Performance Comparison of Collaborative-Filtering Approach with Implicit and Explicit Data
}

\author{
Fitri Marisa*, ${ }^{*}$, Sharifah Sakinah Syed Ahmad ${ }^{2}$, Zeratul Izzah Mohd Yusoh ${ }^{3}$, Tubagus Mohammad Akhriza ${ }^{4}$ \\ Wiwin Purnomowati ${ }^{5}$, Rakesh Kumar Pandey ${ }^{6}$ \\ Informatics Engineering, University of Widyagama, Jl. Borobudur no. 35 Malang, +62-341-482282, Indonesia ${ }^{1}$ \\ Research fellow of Department of Science Technology (DST) under AIRTF Program -RTF/2018/000033 India ${ }^{1}$ \\ Centre for Advanced Computing Technology, Faculty of Information and Communication Technology \\ Universiti Teknikal Malaysia Melaka, Jalan Hang Tuah Jaya, Durian Tunggal, Melaka 76100, Malaysia ${ }^{1,2,3}$ \\ Pradnya Paramita School of Informatics and Computer, J1. LA Sucipto no. 249A Malang, Indonesia ${ }^{4}$ \\ Accounting, University of Widyagama, Jl. Borobudur no. 35 Malang, +62-341-482282, Indonesia ${ }^{5}$ \\ Kirori Mal College - University of Delhi - North campus Delhi 110007- India ${ }^{6}$
}

\begin{abstract}
Challenge in developing a collaborative filtering (CF)-based recommendation system is the problem of coldstarting of items that causes the data to sparse and reduces the accuracy of the recommendations. Therefore, to produce high accuracy a match is needed between the types of data and the approach used. Two approaches in CF include user-based and item-based CFs, both of which can process two types of data; implicit and explicit data. This work aims to find a combination of approaches and data types that produce high accuracy. Cosine-similarity is used to measure the similarity between users and also between items. Mean Absolute Error is also measured to discover the accuracy of a recommendation. Testing of three groups of data based on sparseness results in the best accuracy in an explicit data-based approach that has the smallest MAE value. The result is that the average MAE value for user based (implicit data) is 0.1032 , user based (explicit data) is 0.2320 , item based (implicit data) is 0.3495 , and item based (explicit data) is 0.0926 . The best accuracy is in the item-based (explicit-data) approach which is the smallest average MAE value.
\end{abstract}

Keywords-Recommender system; collaborative-filtering; userbased; item-based; implicit-data; explicit-data

\section{INTRODUCTION}

Mining on data of the small-medium enterprises (SME), is currently needed to improve their progress [1]. Many data mining approaches can be used to explore and utilize the data, such as to make use the data in developing recommendation system (RS). Among the existing aspects, type of data to be processed by RS is an important knowledge to know by the analysts.

RS is defined as an intelligent agent that advises users to find items that are more attractive to them, where users do not need to be involved with data stacks that are not related to their needs [2]. RS can be applied in many fields, for example in e-commerce, RS is useful for recommending items that suit the interests and needs of users; In digital libraries, RS provides recommendations about books or other media that are relevant to user needs [3].

Currently the recommendation system is classified into three: Content-based-filtering, Collaborative-filtering, and hybrid-recommender-systems[4],[5],[6]. One well-known RS approach is the collaborative filtering (CF) method which processes data to produce recommendations based on user's rating preference on the item, where recommendations are generated from implicit or explicit data[7],[8].[9],[10]. The first data type is generated from the flow of transactions that already exist in the system that can be indirectly claimed as customer behavior, such as data on transactions for the purchase of goods, rental of goods; whereas the second type is data generated from planned data collection strategies obtained from users and to be managed in RS. These two types of data certainly have different characters and if processed in a CF approach will produce different results as well [11],[12],[13]. Because suggested objects can be either users or items, CF methods are also developed based on these targets. To simplify, we use terms user-based and item-based $\mathrm{RS}$ to represent these approaches respectively. In user-based $\mathrm{CFs}$, as recommended objects are items, each user is represented as an item vector. Conversely, when recommending users in item-based $\mathrm{CF}$, each item is presented as a user vector. Similarities between users are measured using the cosine similarity metric, as well as measuring the similarity of items.

However, the CF method must overcome the cold-start problem on new items that have not been rated by any user. [14] As a result, the data and hence also the matrix representing the data become sparse and can result in decreased accuracy of the recommendations produced.

This paper discusses the performance comparison of userbased and item-based CFs on implicit and explicit data. The data used in experimental work is a real transaction data taken from SMEs that sell electronic cellular data. The data is divided into three groups based on $10 \%, 30 \%$, and $50 \%$ of rating sparseness in the matrix. Calculations are performed to determine accuracy using an average of Mean Absolute Error (MAE); and as a result, the smallest average MAE value is found in item-based CFs with explicit data that shows the highest level of accuracy.

*Corresponding Authors 


\section{RELATED WORKS}

\section{A. Recommender System}

The RS is designed to help users by proposing options of needed items which can also be presented in the form of personalization to predict user interests and preferences by relying on information-seeking behavior so that they can make the search process effective [2][13]. Some studies categorize Recommender-Systems with different categories. According to [13], RS is categorized as four categories: CF, ContentBased Filtering, Knowledge-Based Filtering, and Hybridbased. In another study, RS is defined based on information gathered and classified into some different types in addition to those mentioned earlier i.e. Demographic based RS, Knowledge based RS, and Utility based RS [5].

\section{B. Collaborative Filtering based Recommender System}

The CF based RS is the earliest and most successful method in RS development and implementation. CF has shown good performance in managing data-sets to extract preference information that is more difficult to find intuitively. It is a method that makes an automatic prediction by filtering user interests by gathering information preferences from the number of collaborating users. One of its advantages over content-based is that $\mathrm{CF}$ can recommend items without additional computing costs or copyright issues involved with processing items directly. Collaborative filtering is generally claimed to be one of the most successful recommendation techniques [7] [15]. There are two types in CollaborativeFiltering: User-based and Item-based [16]. User-based is oriented to user preference data, while Item-based is oriented to product item preferences.

In general, $\mathrm{CF}$ relies on explicit feedback collected directly from users by means of a direct survey presented with a rating model. However, explicit-data has some challenges in difficulty of data collection. It is not easy to get data users who voluntarily give an assessment of the product.[16]. Therefore, implicit-data becomes an alternative to be used in processing data using Collaborative-filtering. The term "implicit" is used here somewhat excessively, so as to express that a user is never actually prompted to express a degree of preference to categories [20]. Implicit data can be generated from customer purchase data taken from transaction data. Researches in [16] [9] and [10] claimed that study of CF method with implicit data, conclude that its performances are depended on the type of data. Hence, it is important to do a performance comparison based on data types.

In addition, cold-start and data sparseness problems are still challenges and weaknesses of this approach. Cold-start problems are caused by new items or users that have never sent or received a previous rating. If a new item appears in the database, there is no way available to recommend it to the user until more information about it has been obtained through another user who assesses or determines other similar items. Sparseness problem is how in general the item-user matrix is rarely filled, making it difficult to identify users and similar items, due to lack of rank and overlapping [14]. Therefore, studies are needed to examine this approach toward data sparseness.

\section{Similarity Calculation in CF based RS}

CF-based approach relies on calculating similarity between users and between items when recommending an object. When recommending items to users, each user is represented as an item vector or user-item matrix; and analogous to that, when recommending users to other users based on items (category), then each item is presented in a user vector or item-user matrix, such as shown in Fig. 1. In this figure, zero means the item is not rated by a user, and one means the vice versa. After becoming vectors, the similarity between users (and also items) can be calculated by measuring distances between vectors using well-known metrics, such as cosinesimilarity and Pearson coefficient correlation. The greater the value of the similarity of the vectors, the items (or users) are seen as more relevant to other users (or items) [17], [18], [19].

Implementation of Pearson coefficient correlation to userbased and item-based CF can be found in [20]. Given the following sets:

- A set of $m$ users $U=\left\{u_{x}: x=1,2, \ldots, m\right\}$,

- A set of $n$ items $I=\left\{i_{x}: x=1,2, \ldots, n\right\}$, and

- A set of $p$ categories $C=\left\{c_{x}: x=1,2, \ldots, p\right\}$.

- A set of $q$ explicit rating $R=\left\{r_{x}: x=1,2, \ldots, q \wedge\right.$ $q \leq m * n\}$

- A set of $t$ implicit rating $R=\left\{r_{x}^{\prime}: x=1,2, \ldots, t \wedge\right.$ $t \leq m * p\}$

As well, the explicit rating of a user $u_{x}$ with reference to an item $i_{h}$ is represented with $r_{u_{x}, i_{h}}$, and the average explicit rating of a user $u_{x}$ as $\overline{r_{u_{x}}}$.

Variables of $K_{x, y}$ and $\lambda_{x, y}$ in (1) and (2) respectively are Pearson coefficient correlation of explicit and implicit data. The item set that is rated together by $u_{x}$ and $u_{y}$ is denoted as $I^{\prime}=\left\{i_{x}: x=1,2,3, \ldots n^{\prime} \wedge n^{\prime} \leq n\right\}$, where $n$ is total number of items. Similarity between $u_{x}$ and $u_{y}$ is denoted as the Pearson correlation coefficient of associated rows of them in the user-item matrix.

$$
\begin{aligned}
& K_{x, y}=\operatorname{sim}\left(u_{x}, u_{y}\right) \\
& =\frac{\sum_{h=1}^{n^{\prime}}\left(r_{u_{x}, i_{h}}-\overline{r_{u_{x}}}\right)-\left(r_{u_{y}, i_{h}}-\overline{r_{u_{y}}}\right)}{\sqrt{\sum_{h=1}^{n^{\prime}}\left(r_{u_{x}, i_{h}}-\overline{r_{u_{x}}}\right)^{2}} \sqrt{\sum_{h=1}^{n^{\prime}}\left(r_{u_{y}, i_{h}}-\overline{r_{u_{y}}}\right)^{2}}}
\end{aligned}
$$

The implicit approach can actually be interpreted as an approach that utilizes the absence of obvious user preferences for an item; by taking advantage that an item consists of several categories so that it is possible to build a categorybased user model. It means that it is possible to infer the preference of a user $u_{x} \in U$ to $c_{x} \in C$ user category by the user-category matrix. This preference can be claimed as an implicit rating $r^{\prime}{ }_{u_{x}, c_{x}} \in R$. Pearson correlation coefficient of the implicit ranking of the two users for all categories $c_{x} \in C$ where $p$ identifies the number of categories notated in (2). 


\begin{tabular}{|l|c|c|c|c|c|c|}
\hline \multicolumn{3}{l}{ USER BASED -CF } & & & \\
\hline user & item 1 & item2 & item3 & item4 & item5 \\
\hline Alice & 1 & 1 & 1 & 1 & 0 \\
\hline User1 & 1 & 1 & 1 & 0 & 1 \\
\hline User2 & 1 & 1 & 0 & 1 & 0 \\
\hline User3 & 0 & 0 & 1 & 1 & 1 \\
\hline & & & & & \\
\hline & & & & & \\
\hline & & & & & \\
\hline ITEM BASED-CF & & & \\
\hline item & alice & user1 & user2 & user3 & \\
\hline item1 & 1 & 1 & 1 & 0 & \\
\hline item2 & 1 & 1 & 1 & 0 & \\
\hline item3 & 1 & 1 & 0 & 1 & \\
\hline item4 & 1 & 0 & 1 & 1 & \\
\hline item5 & 0 & 1 & 0 & 1 & \\
\hline
\end{tabular}

Fig. 1. Items Vector (upper) and users Vector (Lower).

$$
\begin{aligned}
& \lambda_{x, y}=\operatorname{sim}\left(u_{x}, u_{y}\right) \\
& =\frac{\sum_{h=1}^{p}\left(r^{\prime} u_{x}, c_{h}-\overline{r^{\prime} u_{x}}\right)-\left(r^{\prime} u_{y}, c_{h}-\overline{r^{\prime} u_{y}}\right)}{\sqrt{\sum_{h=1}^{p}\left(r^{\prime} u_{x}, c_{h}-\overline{r^{\prime} u_{x}}\right)^{2}} \sqrt{\sum_{h=1}^{p}\left(r^{\prime} u_{y}, c_{h}-\overline{r^{\prime} u_{y}}\right)^{2}}}
\end{aligned}
$$

For item-based approach, formulas in (3) describe Pearson coefficient correlation for explicit data which are represented in variables of $\mu_{x, y}$. Here, the user set that have rated both items $i_{x}$ and $i_{y}$ is denoted as $U^{\prime}=\left\{u_{x}: x=1,2, \ldots, m^{\prime} \wedge\right.$ $\left.m^{\prime} \leq m\right\}$, where $m$ is total number of users. The similarity between $i_{x}$ and $i_{y}$ is denoted as the Pearson correlation coefficient of the associated columns of them in the user-item matrix.

$$
\begin{aligned}
& \mu_{x, y}=\operatorname{sim}\left(i_{x}, i_{y}\right) \\
& =\frac{\sum_{h=1}^{m^{\prime}}\left(r_{u_{h}, i_{x}}-\overline{r_{l x}}\right)-\left(r_{u_{h}, i_{y}}-\overline{r_{l y}}\right)}{\sqrt{\sum_{h=1}^{m^{\prime}}\left(r_{u_{h}, i_{x}}-\bar{r}_{l x}\right)^{2}} \sqrt{\sum_{h=1}^{m^{\prime}}\left(r_{u_{h}, i_{y}}-\overline{r_{l y}}\right)^{2}}}
\end{aligned}
$$

Formula (4) applies when we use item-category bitmap matrix, where $p$ is the number of categories and $v_{u_{h}, i_{x}}$ is a Boolean value of value which equal to 1 provided that item $x$ is owned by category $h$ or equal to 0 otherwise.

$$
\begin{aligned}
& v_{x, y}=\operatorname{sim}\left(i_{x}, i_{y}\right) \\
& =\frac{\sum_{h=1}^{p}\left(v_{c_{h}, i_{x}}-\overline{v_{l x}}\right)-\left(v_{c_{h}, i_{y}}-\overline{v_{l y}}\right)}{\sqrt{\sum_{h=1}^{p}\left(v_{u_{h}, i_{x}}-\overline{v_{l x}}\right)^{2}} \sqrt{\sum_{h=1}^{p}\left(v_{u_{h}, i_{y}}-\overline{v_{l y}}\right)^{2}}}
\end{aligned}
$$

\section{Prediction and Recommendation Values}

For the User-based approach, the closest neighbor subset user is selected based on its similarity to the active user. The steps are to find the closest neighbor user with the greatest similarity value, then calculate the predicted value of items that have been selected by the nearest neighbor user but have never been chosen by an active user. Supposed $a$ is an active user being examined, $p$ is the item whose prediction is calculated, and $r_{u}$ has $a$ value of ' 1 ', indicating that the closest neighbor user $u$ has chosen item $p$. The prediction value of user $a$ with respect to item $p$ is calculated using (5).

$\operatorname{pred}(a, p)=r_{u} * \operatorname{sim}(a, u)$

For Item-based approach, the nearest neighbor subset item is selected based on their similarity to items that have been selected by the active user. The steps are to find the nearest neighbor item with the greatest similarity value, then calculate the predicted value of the closest neighbor items that have never been selected by an active user based on its similarity to items that have been selected by an active user.

$\operatorname{pred}(a, p)=r_{a} * \operatorname{sim}(i, p)$

The prediction value calculated using (6), where $r_{a}$ has $a$ value of ' 1 ', indicating that the active user has chosen item $i$.

\section{E. Mean Absolute Error (MAE)}

MAE, also known as Mean Median Regression [20],[21], is used to measure the accuracy of forecasting models [22]. Based on the formula, a small error value will be proven with a small MAE value and a large error value will be proven with a greater MAE value. The formula is defined in (7):

MAE $=\sum_{i=1}^{N} \frac{\left|p_{i}-q_{i}\right|}{N}$

Where $p_{i}=$ value of $i, q_{i}=$ prediction of $\mathrm{i}$, and $\mathrm{N}=$ number of records in the given data.

\section{RESEARCH METHODOLOGY}

The proposed model for comparing the performance of the $\mathrm{CF}$ approach is given in Fig. 2 with the explanation as follows.

1) Preparing and determining normalization of implicit and explicit data.

2) Calculating the similarity with pearson coefficien correlation for each type of dataset based on formulas as defined in (1), (2), (3) and (4).

3) Calculating the prediction value for each type of dataset using (5),(6).

4) Calculating MAE for each dataset using (7).

5) Calculating the average of MAE.

6) Comparing four MAE values: User-based with implicit data, Item-based with implicit data, User-based with explicit data, and Item-based with explicit data. 


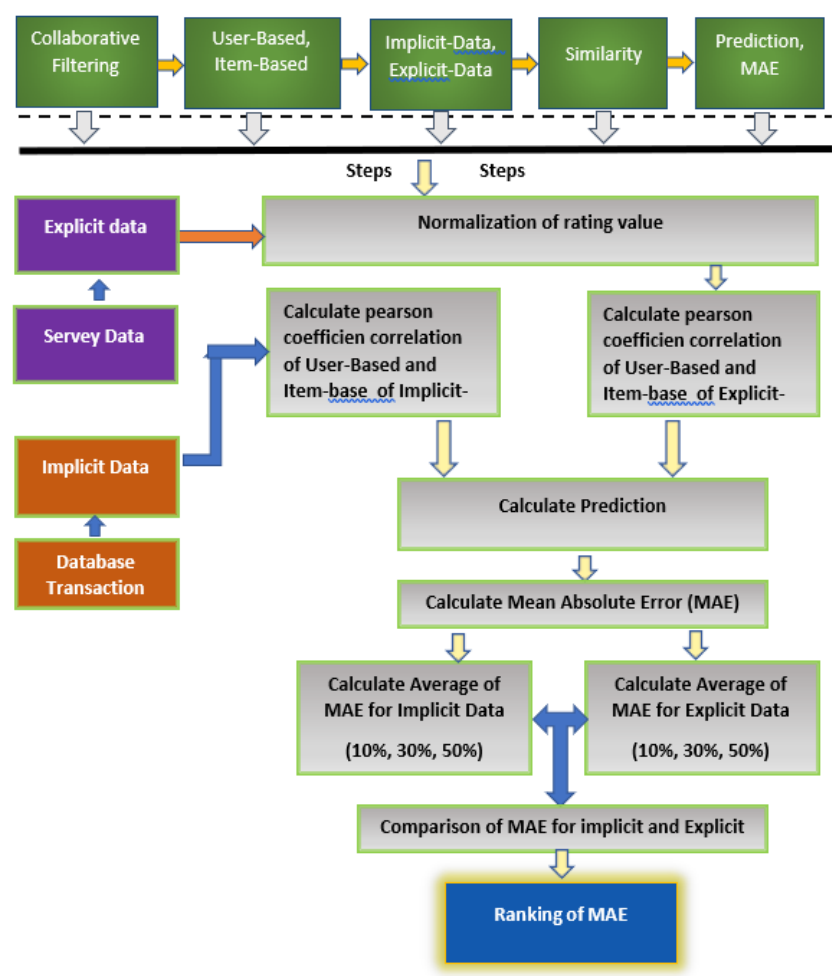

Fig. 2. Model of Comparison of Performance in Collaborative-Filtering Type [23],[24].

\section{EXPERIMENTAL RESEARCH}

The results of this experiment are the presentation of MAE results with the process steps described in Fig. 3. The steps taken are calculating similarity with the Pearson coefficient correlation, calculating predictions, and calculating MAE.

\section{A. Performance of User-based with Implicit-Data}

Based on the test results on the sample data of user-based matrics, the data has been grouped based on the number of random ratings vacancies in three groups namely $10 \%, 30 \%$, and $50 \%$. Then each group was calculated similarity, prediction, and MAE. the results of the test have been described in Table I, and illustrated in the graph (Fig. 4).

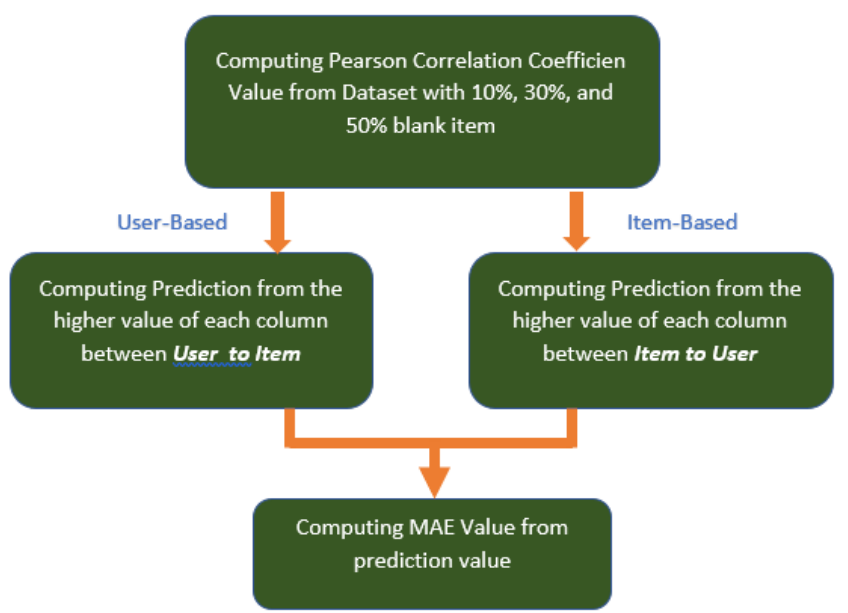

Fig. 3. Process to Compute Similarity with Pearson Correlation, Prediction, and MAE [12],[23].
TABLE. I. COMPARISON OF MAE IN USER-BASED WITH IMPLICIT DATA

\begin{tabular}{|l|l|}
\hline Blank Rating & MAE \\
\hline $10 \%$ & 0 \\
\hline $30 \%$ & 0.066277635 \\
\hline $50 \%$ & 0.243543221 \\
\hline Average of MAE & 0.103273619 \\
\hline
\end{tabular}

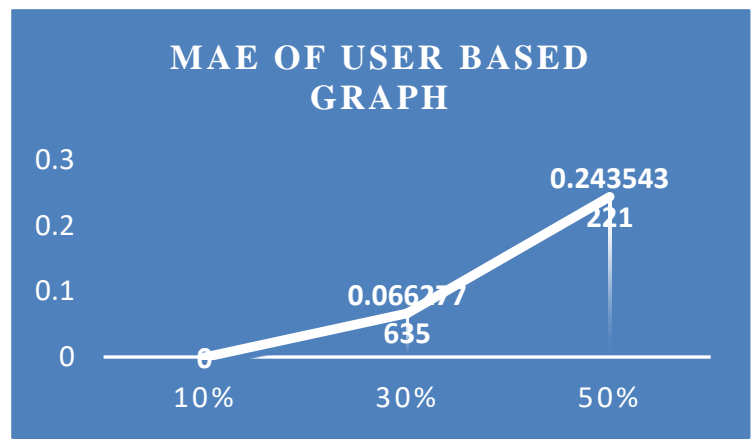

Fig. 4. Graph of MAE Comparison in user-based with Implicit-Data.

The lowest MAE value $=0$ is in the dataset with a blank rating of $10 \%$. It can be concluded that in a user-based Collaborative-Filtering approach involving implicit data, the smaller the value of the blank rating, the more accurate the results are evidenced by the small MAE value.

\section{B. Performance of Item-based with Implicit-Data}

Based on the experiment on the sample data of item-based matrics, the data has been grouped based on the number of random ratings vacancies in three groups namely $10 \%, 30 \%$, and 50\%. Then each group was calculated similarity, prediction, and MAE. The results of the test have been described in Table II and illustrated in the graph (Fig. 5).

TABLE. II. COMPARISON OF MAE IN ITEM-BASED WITH IMPLICIT DATA

\begin{tabular}{|l|l|}
\hline Blank rating & MAE \\
\hline $10 \%$ & 0 \\
\hline $30 \%$ & 0.048660458 \\
\hline $50 \%$ & 1 \\
\hline Average & 0.349553486 \\
\hline
\end{tabular}

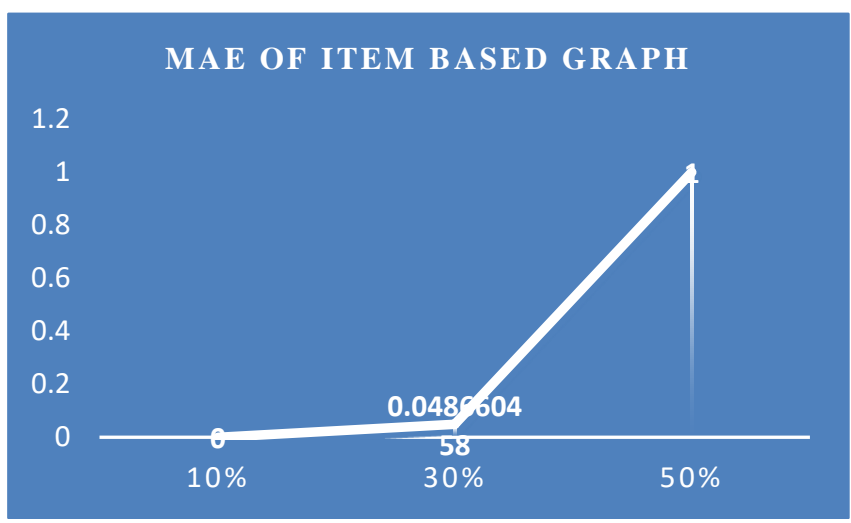

Fig. 5. Graph of MAE Comparison in Item-based with Implicit-Data. 
The lowest MAE value $=0$ is in the dataset with a blank rating of $10 \%$. It can be concluded that in an item-based Collaborative-Filtering approach involving implicit data, the smaller the value of the blank rating, the more accurate the results are evidenced by the small MAE value.

\section{Comparison between User-Based and Item-based use Implicit Data}

Table III and Fig. 6 have described the comparison of MAE values between user-based and item-based that involve implicit data. The smallest MAE value generated from this test is a user-based CF approach with a value of 0.103 which means a user-based CF approach that involves implicit data is more accurate than an item-based CF approach involving the same type of data.

\section{Performance of User-Based with Explicit Data}

Based on the test results on the sample data of user-based metrics, the data has been grouped based on the number of random ratings vacancies in three groups namely $10 \%, 30 \%$, and $50 \%$. Then each group was calculated similarity, prediction, and MAE. The results of the test have been described in Table IV, and illustrated in the graph (Fig. 7).

TABLE. III. COMPARISON OF MAE BETWEEN USER-BASED AND ITEMBASED USE IMPLICIT DATA

\begin{tabular}{|l|l|l|}
\hline Blank rating & User-based & Item-Based \\
\hline $10 \%$ & 0 & 0 \\
\hline $30 \%$ & 0.066278 & 0.04866 \\
\hline $50 \%$ & 0.243543 & 1 \\
\hline Average & 0.103274 & 0.349553 \\
\hline
\end{tabular}

MAE Comparison of User Based and Item based use Implicit data

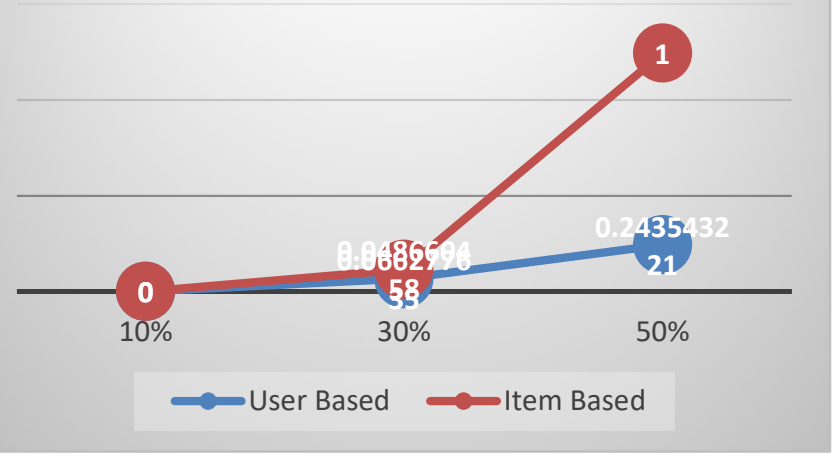

Fig. 6. Graph of MAE Comparison in Item-based and user-based in ImplicitData.

TABLE. IV. COMPARISON OF MAE IN USER-BASED WITH EXPLICIT- DATA

\begin{tabular}{|l|l|}
\hline Blank rating & MAE \\
\hline $10 \%$ & 0.425972049 \\
\hline $30 \%$ & 0.054072837 \\
\hline $50 \%$ & 0.216221325 \\
\hline Average & 0.232088737 \\
\hline
\end{tabular}

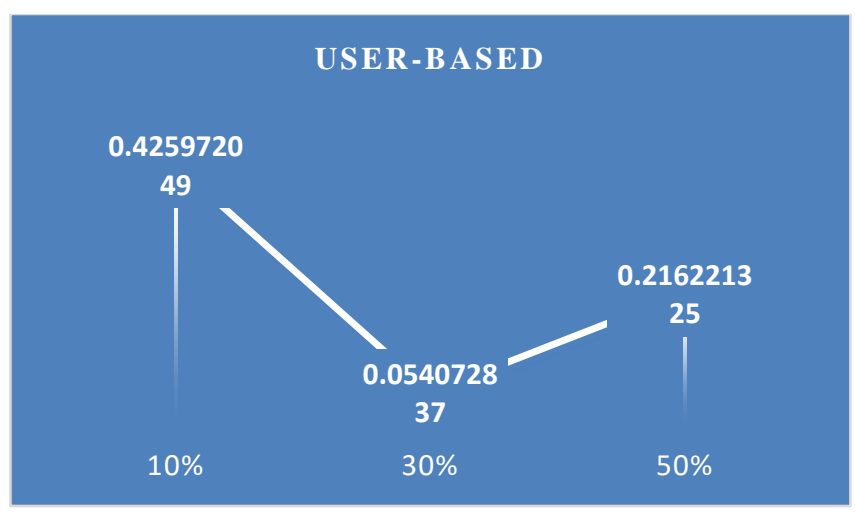

Fig. 7. Graph of MAE Comparison in user-based with Explicit-Data.

The lowest MAE value $=0.054$ is in the dataset with a blank rating of $30 \%$. It can be concluded that the smallest MAE value is unpredictable and cannot be identified by the least amount of rating blanks as well. And this has shown differences in results compared to testing involving implicit data.

\section{E. Performance of Item-Based with Explicit Data}

Based on the experiment on the sample data of item-based metrics, the data has been grouped based on the number of random ratings vacancies in three groups namely $10 \%, 30 \%$, and $50 \%$. Then each group was calculated similarity, prediction, and MAE. The results of the test have been described in Table V, and illustrated in the graph (Fig. 8).

The lowest MAE value $=0.0450$ is in the dataset with a blank rating of $10 \%$. It can be concluded that in an item-based Collaborative-Filtering approach involving explicit data, the smaller the value of the blank rating, the more accurate the results are evidenced by the small MAE value.

TABLE. V. COMPARISON OF MAE IN ITEM-BASED WITH EXPLICIT- DATA

\begin{tabular}{|l|l|}
\hline Blank rating & MAE \\
\hline $10 \%$ & 0.045066008 \\
\hline $30 \%$ & 0.095425989 \\
\hline $50 \%$ & 0.13727043 \\
\hline Average & 0.092587476 \\
\hline
\end{tabular}

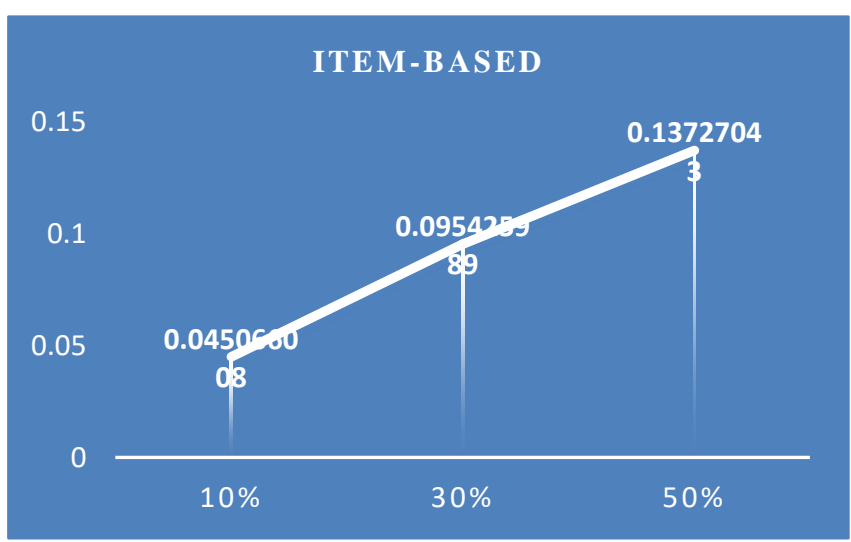

Fig. 8. Graph of MAE Comparison in Item-based with Explicit-Data. 


\section{F. Comparison between of User-Based and Item-Based use Explicit Data}

Table VI and Fig. 9 have described the comparison of MAE values between user-based and item-based that involve explicit data. The smallest MAE value generated from this test is an item-based CF approach with a value of 0.092 which means an item-based $\mathrm{CF}$ approach that involves explicit data is more accurate than user-based $\mathrm{CF}$ approach involving the same type of data.

\section{G. Comparison of MAE in All Types and Data}

The final step is to compare the MAE values of the four experimental groups, which are described in Table VII and Fig. 10. From this study, the best accuracy is that the itembased CF approach involves explicit data, as evidenced by the smallest MAE value (0.0926).

TABLE. VI. COMPARISON OF MAE IN EXPLICIT-DATA

\begin{tabular}{|l|l|l|}
\hline Blank rating & MAE of User-based & MAE of Item-Based \\
\hline $10 \%$ & 0.425972 & 0.045066 \\
\hline $30 \%$ & 0.054073 & 0.095426 \\
\hline $50 \%$ & 0.216221 & 0.13727 \\
\hline Average & 0.232089 & 0.092587 \\
\hline
\end{tabular}

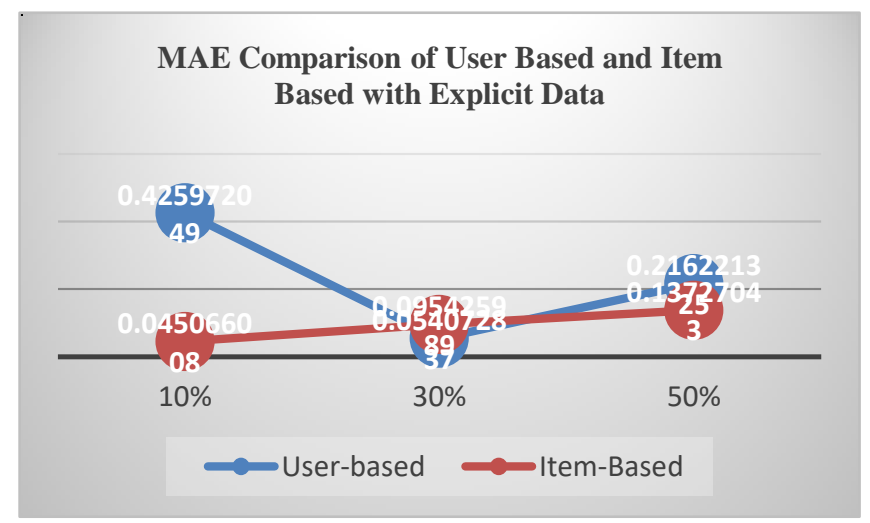

Fig. 9. Graph of MAE Comparison in Item-based and user-based in ExplicitData.

TABLE. VII. COMPARISON OF MAE IN ALL TYPE AND DATA

\begin{tabular}{|l|l|l|l|l|}
\hline Blank Rating & UB-Boolean & IB-Boolean & UB-Nom & IB-Nom \\
\hline $10 \%$ & 0 & 0 & 0.426 & 0.0451 \\
\hline $30 \%$ & 0.066 & 0.0487 & 0.0541 & 0.0954 \\
\hline $50 \%$ & 0.244 & 1 & 0.2162 & 0.1373 \\
\hline Average & 0.103 & 0.3496 & 0.2321 & 0.0926 \\
\hline
\end{tabular}

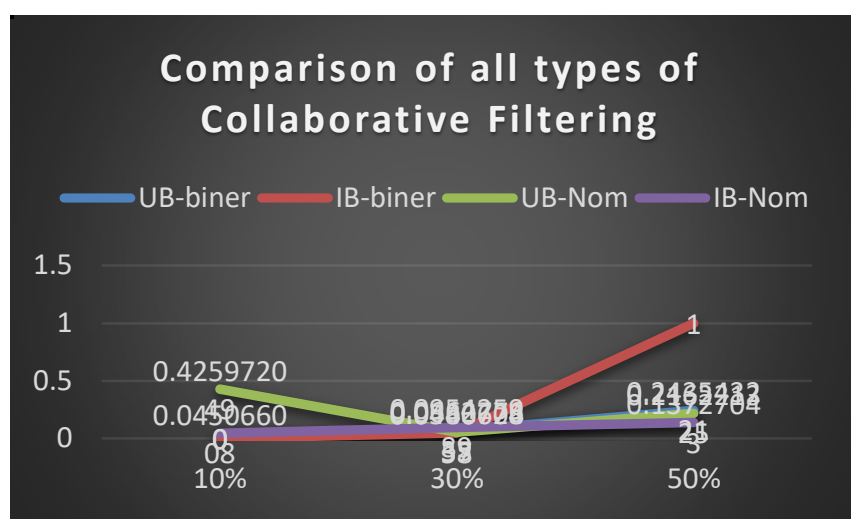

Fig. 10. Graph of Comparison all types of Collaborative Filtering.

\section{CONCLUSION AND FUTURE WORK}

The results of this study are Comparison models of User Based CF and Item Based CF performance involving Implicit and Explicit data.

User-Based CF with Implicit Data produces the smallest MAE value of 0 , in the lowest empty-rating dataset (10\%). It can be concluded that the smaller the number of blank ratings in the dataset, the more accurate the recommendations it produces.

Item-Based CF with Implicit Data produces the smallest MAE value of 0 , in the lowest empty-rating dataset (10\%). It can be concluded that the smaller the number of blank ratings in the dataset, the more accurate the recommendations it produces.

The average MAE value of the User-Based CF approach is 0.103, which is smaller than the item-based CF approach. It can be concluded that User Based CF performance is more accurate for processing implicit data than Item Based.

The User-Based CF Approach with Explicit Data yields the smallest MAE value of 0.054 in the blank rating dataset group $(30 \%)$. It can be concluded that the number of blank ratings in the Explicit data applying the User Based $\mathrm{CF}$ approach cannot predict the level of accuracy of the resulting recommendation recommendations.

Item-Based CF with explicit Data produces the smallest MAE value of 0.045 , in the lowest empty-rating dataset $(10 \%)$. It can be concluded that the smaller the number of blank ratings in the dataset, the more accurate the recommendations it produces.

The average MAE value of the Item-Based CF approach is 0.092, which is smaller than the user-based CF approach. It can be concluded that Item Based CF performance is more accurate for processing explicit data than user Based CF. 
Based on the four approaches tested, the smallest average MAE is Item-Based CF with Explicit-Data. So based on this research, the highest level of accuracy performance for Collaborative-Filtering is the Item-Based CF approach with explicit data.

\section{ACKNOWLEDGMENT}

We would like to thank all those who contributed to this research starting from the Universiti Teknikal Malaysia Melaka (UTem); Malaysia, DRPM of Ministry of Research and Technology \& Higher Education, Indonesia; Widyagama University Malang, Indonesia; Department of Science and Technology (DST) under AIRFT India (RTF / RTF / 2018/000033); AR-PulsaBiz Reload Malang, Indonesia which is one of the SMEs in Indonesia; and last but not least, all reviewers who provided some valuable criticisms and suggestions to improve this paper quality.

\section{REFERENCES}

[1] F. Marisa et al., "Segmentation Model of Customer Lifetime Value in Small and Medium Enterprise ( SMEs ) using K-Means Clustering and LRFM Model," Int. J. Integr. Eng., vol. 11, no. 3, pp. 169-180, 2019.

[2] S. Ayyaz, U. Qamar, and R. Nawaz, "HCF-CRS: A Hybrid content based fuzzy conformal recommender system for providing recommendations with confidence," PLoS One, vol. 13, no. 10, pp. 130, 2018.

[3] R. Burke, "Hybrid Web Recommender Systems," Adapt. Web, pp. 377408, 2007.

[4] Y. Cai, H. F. Leung, Q. Li, H. Min, J. Tang, and J. Li, "Typicality-based collaborative filtering recommendation," IEEE Trans. Knowl. Data Eng., vol. 26, no. 3, pp. 766-779, 2014.

[5] R. Burke, “A Knowledge Based Recommender System," Int. J. Comput. Intell. Syst., vol. 1, no. 3, pp. 1-23, 2008.

[6] Y. A. Pratama, D. Wijaya, and A. Halim, "Digital Cakery Dengan Algoritma Collaborative Filtering," JSM STMIK Mikroskil, vol. 14, no. 1, pp. 79-88, 2013.

[7] T. Osadchiy, I. Poliakov, P. Olivier, M. Rowland, and E. Foster, "Recommender system based on pairwise association rules," Expert Syst. Appl., vol. 115, pp. 535-542, 2019.

[8] J. Chen, C. Miller, and G. G. Dagher, "Product recommendation system for small online retailers using association rules mining," Proc. 2014 Int. Conf. Innov. Des. Manuf. ICIDM 2014, vol. 17, pp. 71-77, 2014.

[9] M. Prasasti, R. Saptono, and Winarno, "Sistem Rekomendasi Peminjaman Buku di UPT Perpustakaan UNS dengan Metode Itembased Collaborative Filtering dan Rating Implisit," vol. 1, pp. 280-287, 2017.
[10] K. Choi, D. Yoo, G. Kim, and Y. Suh, "A hybrid online-product recommendation system: Combining implicit rating-based collaborative filtering and sequential pattern analysis," Electron. Commer. Res. Appl., vol. 11, no. 4, pp. 309-317, 2012.

[11] L. Dzumiroh and R. Saptono, "Penerapan Metode Collaborative Filtering Menggunakan Rating Implisit pada Sistem Perekomendasi Pemilihan Film di Rental VCD,” J. Teknol. Inf. ITSmart, vol. 1, no. 2, p. 54, 2016.

[12] M. K. Najafabadi, M. N. ri Mahrin, S. Chuprat, and H. M. Sarkan, "Improving the accuracy of collaborative filtering recommendations using clustering and association rules mining on implicit data," Comput. Human Behav., vol. 67, pp. 113-128, 2017.

[13] Z. Dehghani and H. R. Jamali, "A multi-layer contextual model for recommender systems in digital libraries," in Aslib Proceedings, 2011, vol. 63, no. 6, pp. 555-569.

[14] Y. S. Kim and Y. S. Kim, "Text recommender system using user' s usage patterns," 2011.

[15] G. Li, Y. Chen, Z. Zhang, J. Zhong, and Q. Chen, "Social personalized ranking with both the explicit and implicit influence of user trust and of item ratings," Eng. Appl. Artif. Intell., vol. 67, no. August 2017, pp. 283-295, 2018.

[16] T. Q. Lee, Y. Park, and Y. T. Park, "A time-based approach to effective recommender systems using implicit feedback," Expert Syst. Appl., vol. 34, no. 4, pp. 3055-3062, 2008.

[17] E. Setiawan and A. Muttaqin, "Implementation of K-Nearest Neightbors Face Recognition on Low-power Processor," TELKOMNIKA (Telecommunication Comput. Electron. Control., vol. 13, no. 3, p. 949, 2016.

[18] M. Yusril, H. Setyawan, R. M. Awangga, and N. A. Lestari, "K-Nearest neighbor algorithm on implicit feedback to determine SOP," TELKOMNIMA, vol. 17, no. 3, p. 12928, 2019.

[19] H. Kalhori, M. M. Alamdari, and L. Ye, "Automated algorithm for impact force identification using cosine similarity searching," Meas. J. Int. Meas. Confed., vol. 122, no. July 2017, pp. 648-657, 2018.

[20] A. de Myttenaere, B. Golden, B. Le Grand, and F. Rossi, "Mean Absolute Percentage Error for regression models," Neurocomputing, vol. 192, pp. 38-48, 2016.

[21] Kenji Paskal, "Peningkatan Akurasi Metode User Based Collaborative Filtering Menggunakan Agglomerative Clustering pada Sistem Rekomendasi," UNIVERSITAS SANATA DHARMA, 2018.

[22] P. H. Franses, "A note on the Mean Absolute Scaled Error," Int. J. Forecast., vol. 32, no. 1, pp. 20-22, 2016.

[23] M. Papagelis and D. Plexousakis, "Qualitative analysis of user-based and item-based prediction algorithms for recommendation agents," Eng. Appl. Artif. Intell., vol. 18, no. 7, pp. 781-789, 2005.

T. Badriyah, I. Restuningtyas, and F. Setyorini, "Sistem Rekomendasi Collaborative Filtering Berbasis User Algoritma Adjusted Cosine Similarity," 2017. 